\title{
Ensaios MSCR segundo as normas ASTM D7405-10a e AASHTO T350-14: um estudo de caso envolvendo ligantes asfálticos modificados
}

\author{
Matheus David Inocente Domingos ${ }^{1}$ e Adalberto Leandro Faxina ${ }^{2}$
}

\begin{abstract}
Resumo: Este trabalho tem por objetivo comparar os resultados dos ensaios de fluência e recuperação sob tensão múltipla (MSCR) segundo os protocolos da ASTM D7405-10a e da AASHTO T350-14. A comparação foi feita com base em ensaios realizados com ligantes asfálticos modificados com ácido polifosfórico, borracha moída de pneus e uma combinação de ambos. Os percentuais de recuperação $(\mathrm{R})$ e as compliâncias não-recuperáveis $\left(\mathrm{J}_{\mathrm{nr}}\right)$ foram calculados e as variações foram quantificadas estatisticamente. O protocolo da ASTM subestimou os valores de R e superestimou os valores de $\mathrm{J}_{\mathrm{nr}}$ para todas as formulações, em especial o CAP+borracha e o CAP+borracha+PPA. O CAP+PPA apresentou baixa sensibilidade à tensão e os melhores resultados para $\mathrm{R}$ e $\mathrm{J}_{\mathrm{nr}}$ a $3.200 \mathrm{~Pa}$, em ambos os protocolos, o que justifica a sua escolha como a melhor formulação. Os resultados obtidos com os ligantes asfálticos modificados serviram para ilustrar a importância de se migrar do protocolo da ASTM para o da AASHTO.
\end{abstract}

Palavras-chave: borracha moída de pneus, ácido polifosfórico, ensaio MSCR, grau de desempenho, normas ASTM/AASHTO.

\begin{abstract}
This paper mainly aims at comparing the results of the multiple stress creep and recovery (MSCR) tests according to the ASTM D7405-10a with the ones collected in accordance with the AASHTO T350-14 protocol. These comparisons were made based on tests with asphalt binders modified with polyphosphoric acid, crumb rubber and a combination of both additives. The percent recoveries $(\mathrm{R})$ and the nonrecoverable compliances $\left(\mathrm{J}_{\mathrm{nr}}\right)$ were calculated and their variations were statistically evaluated. The ASTM procedure underestimated the $\mathrm{R}$ values and overestimated the $\mathrm{J}_{\mathrm{nr}}$ values for all the formulations, especially the AC+rubber and the AC+rubber+PPA. The AC+PPA showed low stress sensitivity and the best results for the parameters $\mathrm{R}$ and $\mathrm{J}_{\mathrm{nr}}$ at 3,200 Pa either in the ASTM or the AASHTO protocols, which justifies its choice as the best formulation. The data from the modified asphalt binders suggest that the change from the ASTM protocol to the AASHTO one is recommended.
\end{abstract}

Keywords: crumb rubber, polyphosphoric acid, MSCR test, performance grade, ASTM/AASHTO standards.

\section{INTRODUÇÃO}

A deformação permanente, também conhecida no linguajar popular como "trilha de roda", é um mecanismo de ruptura comumente encontrado em pavimentos asfálticos sujeitos a tráfego pesado, sendo também potencializado por velocidades médias baixas e predominância de veículos lentos na faixa de rolamento (e. g., caminhões e ônibus). As deformações viscosas oriundas da aplicação das cargas após a passagem de cada veículo se acumulam e, após um determinado momento, passam a ser significativas e visíveis. Este tipo de defeito se origina do acúmulo de deformações plásticas em uma ou mais camadas do pavimento (revestimento, base, sub-base e reforço de subleito) e no subleito. Em nível laboratorial, o estudo da deformação permanente em misturas asfálticas é geralmente feito por meio de simuladores de tráfego (D’Angelo et al., 2007; Kim et al., 2009; Moura, 2010; Reinke, 2010; FHWA, 2011; Wasage et al., 2011; Gibson et al., 2012) e de ensaios de fluência e recuperação, também chamados de flow number (Witczak et al., 2002; Nascimento, 2008; Wasage et al., 2009; Onofre, 2012; Onofre et al., 2013; Apeagyei, 2014). Outros métodos como o monitoramento de pistas experimentais (Moura,

\footnotetext{
1 Matheus David Inocente Domingos, Departamento de Engenharia de

Transportes, EESC-USP. (matheusdavid@sc.usp.br)

2 Adalberto Leandro Faxina, Departamento de Engenharia de

Transportes, EESC-USP. (alfaxina@sc.usp.br)
}

Manuscrito recebido em 05/04/2016 e aprovado para publicação em 28/10/2016

Este artigo é parte de TRANSPORTES v. 24, n. 3, 2016. ISSN: 2237-1346 (online). DOI:10.4237/transportes.v24i3.1115
2010) e o uso de ensaios triaxiais (Witczak et al., 2002; Borges, 2014) e de módulo dinâmico (Witczak et al., 2002; Li et al., 2011) também podem ser encontrados na literatura.

Com relação ao ligante asfáltico, diversos procedimentos de ensaio e parâmetros têm sido sugeridos pelos pesquisadores para verificar a sua suscetibilidade à deformação permanente. Juntamente com a publicação da especificação Superpave ${ }^{\circledR}$ em meados dos anos 90 , o parâmetro $\mathrm{G}^{*} / \operatorname{sen} \delta$ (magnitude do módulo complexo $\mathrm{G}^{*}$ dividida pelo seno do ângulo de fase $\delta$ ) foi sugerido como o critério oficial para quantificação da suscetibilidade do CAP ao acúmulo de deformação. A equação deste parâmetro, que é matematicamente igual ao inverso da compliância de dissipação J" (loss compliance) conforme Equação (1), é baseada no conceito de energia dissipada em um ciclo oscilatório de cisalhamento e estabelece que um material é tanto mais resistente à deformação permanente quanto maior for o valor numérico de $\mathrm{G}^{*} / \operatorname{sen} \delta$ (Roberts et al., 1996).

$$
\frac{1}{J^{\prime \prime}}=\frac{G^{*}}{\operatorname{sen} \delta}
$$

em que: $J^{\prime \prime}$ : compliância de dissipação $\left[\mathrm{Pa}^{-1}, \mathrm{kPa}^{-1}\right]$;

$G^{*}$ : magnitude do módulo complexo de cisalhamento [Pa, $\mathrm{kPa}]$; e

$\delta$ : ângulo de fase [graus, rad].

Apesar dos avanços conquistados com o parâmetro $\mathrm{G}^{*} / \operatorname{sen} \delta$ em relação às propriedades tradicionais dos ligantes asfálticos ou Cimentos Asfálticos de Petróleo (CAP), ele não foi aceito devido às suas diversas limitações científicas e à falta de correlação com os resultados de ensaios de misturas asfálticas. Tais limitações abrangem (i) a aplicação de poucos ciclos de carregamento; (ii) as medições na faixa de 
viscoelasticidade linear; (iii) o impacto relativamente pequeno da elasticidade da formulação $(\delta)$ no resultado final do parâmetro; e (iv) a incapacidade do ensaio oscilatório em distinguir claramente os desempenhos de todos os ligantes asfálticos modificados quanto à deformação permanente (Bahia et al., 2001; Delgadillo et al., 2006a, 2006b; DuBois et al., 2014).

Dentre as alternativas propostas pelos pesquisadores para substituir ou refinar o parâmetro $\mathrm{G}^{*} / \operatorname{sen} \delta$, a aplicação de ciclos sucessivos de carregamento e descarregamento em uma amostra de CAP - tal como já era feito há algum tempo em procedimentos com misturas asfálticas - foi bem vista na literatura como uma alternativa ao ensaio em regime oscilatório (Bahia et al., 2001; Delgadillo et al., 2006a, 2006b; D'Angelo et al., 2007). Como uma consequência destas observações, protocolos foram desenvolvidos e publicados em estudos científicos. O primeiro deles, designado como RCRT (repeated creep and recovery test, ou "ensaio de fluência repetida e recuperação"), estabelecia que uma amostra de CAP condicionada entre as duas placas paralelas do reômetro de cisalhamento dinâmico (DSR) deveria ser submetida a uma tensão entre 30 e 300 Pa durante 100 ciclos de fluência e de recuperação (Bahia et al., 2001; Delgadillo et al., 2006b). Trabalhos conduzidos por Delgadillo et al. (2006a) incorporaram níveis adicionais de tensão ao RCRT - desde 25 até $10.000 \mathrm{~Pa}$ - para, dentre outros objetivos, verificar a tensão mais adequada para caracterização da resistência do CAP à deformação permanente em um pavimento real. O RCRT foi então refinado e designado como MSCR, sigla para multiple stress creep and recovery ou "fluência e recuperação sob tensão múltipla". O protocolo original do MSCR, descrito por D'Angelo et al. (2007), recomendava a utilização de 11 níveis crescentes de tensão - 25, 50, 100, 200, 400, 800, 1.600, 3.200, 6.400, 12.800 e $25.600 \mathrm{~Pa}-\mathrm{e}$ um total de 10 ciclos de fluência e recuperação em cada nível, sendo $1 \mathrm{~s}$ de fluência e $9 \mathrm{~s}$ de recuperação por ciclo.

Para fins de especificação do MSCR como um ensaio de caracterização e de classificação de ligantes asfálticos na especificação Superpave ${ }^{\circledR}$, as agências norte-americanas AASHTO e ASTM decidiram que apenas dois dos 11 valores de tensão ( 100 e $3.200 \mathrm{~Pa}$ ) seriam mantidos e que, inicialmente, os 10 ciclos de fluência e de recuperação permaneceriam nos protocolos oficiais do MSCR (AASHTO TP70/T350 e ASTM D7405). Alguns anos após a publicação das primeiras normas do MSCR pela ASTM (2008) e AASHTO (2009), atualizações foram feitas com o propósito de, por exemplo, estabelecer percentuais máximos de variabilidade para os resultados do parâmetro $\mathrm{J}_{\mathrm{nr}}$ (compliância não-recuperável). Este foi o caso da norma da ASTM, que não apresentava limites máximos para tal variabilidade nas primeiras versões (D7405-08, D7405-08a e D7405-10) e passou a apresentá-los apenas nas últimas versões
(D7405-10a e D7405-15). No caso da norma da AASHTO, houve quatro atualizações (TP70-10, TP70-12, TP70-13 e T350-14) e uma mudança de designação (de TP70 para T350) em um intervalo de tempo de cinco anos. Entretanto, as mudanças mais radicais ocorreram nas últimas versões, em que as diferenças entre os procedimentos do MSCR segundo a AASHTO e a ASTM passaram a ser significativas. A Tabela 1 mostra as semelhanças e diferenças entre duas destas especificações.

Como se pode observar, a versão mais recente da norma AASHTO TP70 apresenta muitas diferenças em relação à norma correspondente ASTM D7405. Em síntese, estas mudanças incluem (i) o aumento do número de ciclos de fluência e recuperação a $100 \mathrm{~Pa}$; (ii) a desconsideração dos primeiros dez ciclos de creep-recovery nos cálculos dos parâmetros R e $\mathrm{J}_{\mathrm{nr}}$ a $100 \mathrm{~Pa}$; e (iii) o estabelecimento de um critério para verificação do nível de elasticidade do ligante asfáltico (alto ou baixo) em função dos seus resultados de $\mathrm{R}$ e $\mathrm{J}_{\mathrm{nr}}$ a 3.200 Pa. Este critério da elasticidade foi inicialmente publicado nas formas gráfica (Anderson e Bukowski, 2012) e de tabela (FHWA, 2011) como uma verificação complementar da presença de redes poliméricas capazes de proporcionar respostas elásticas altas em ligantes asfálticos com valores de $\mathrm{J}_{\mathrm{nr}} \leq 2,0 \mathrm{kPa}^{-1}$. Com a alteração do status da norma do MSCR de temporária (provisional standard TP70) para definitiva (full standard T350) em 2014, este apêndice foi suprimido do protocolo de ensaio. Entretanto, os outros itens constantes na Tabela 1 se mantiveram semelhantes para as duas normas. Cumpre lembrar também que, com a atualização da norma em 2016 (D7405-15), o protocolo da ASTM ficou igual ao da AASHTO em termos do número de ciclos a $100 \mathrm{~Pa}$ e dos cálculos dos parâmetros $\mathrm{R}$ e $\mathrm{J}_{\mathrm{nr}}$ em ambas as tensões.

A aplicação de um número grande de ciclos de creeprecovery em tensões baixas já era uma prerrogativa na literatura desde as primeiras publicações sobre o RCRT (Bahia et al., 2001; Marasteanu et al., 2005; Delgadillo et al., 2006a, 2006b), pois se desejava alcançar um estado chamado de steady state. Em poucas palavras, este estado designa o comportamento no qual a taxa de deformação acumulada na amostra é constante de um ciclo para outro e independente do nível de deformação atual. Isto significa que, no steady state, a elasticidade retardada não mais exerce influência na resposta fluência-recuperação do ligante asfáltico e o aumento de deformação entre dois ciclos consecutivos é previsível (Golalipour, 2011). Embora a norma AASHTO T350-14 tenha adotado 20 ciclos de creep-recovery a $100 \mathrm{~Pa}$, não existe um consenso entre os pesquisadores de que este número seja suficiente para atingir o steady state em todos os ligantes asfálticos modificados: Bahia et al. (2001) recomendam um mínimo de 50 ciclos, enquanto que Golalipour (2011) e Delgadillo et al. (2006b) sugerem

Tabela 1. Comparações entre as normas do ensaio MSCR (AASHTO, 2013; ASTM, 2010)

\begin{tabular}{|c|c|c|}
\hline Descrição do item & ASTM D7405-10a & AASHTO TP70-13 \\
\hline Número de ciclos de fluência e recuperação a $100 \mathrm{~Pa}$ & 10 & 20 \\
\hline Número de ciclos de fluência e recuperação a $3.200 \mathrm{~Pa}$ & 10 & 10 \\
\hline Diâmetro da amostra/gap (mm) & $25 / 1,0$ & $25 / 1,0$ \\
\hline Principais parâmetros calculados* & $\mathrm{R}, \mathrm{J}_{\mathrm{nr}}$ e $\mathrm{J}_{\mathrm{nr} \text {, diff }}$ & $\mathrm{R}, \mathrm{J}_{\mathrm{nr}}$ e $\mathrm{J}_{\mathrm{nr} \text {, diff }}$ \\
\hline Ciclos utilizados nos cálculos de $\mathrm{R}$ e $\mathrm{J}_{\mathrm{nr}}$ a 100 e a $3.200 \mathrm{~Pa}$ & Todos os ciclos & Apenas os últimos dez ciclos \\
\hline Verificação do nível de elasticidade do ligante asfáltico & $\begin{array}{c}\text { Não apresenta diretrizes para } \\
\text { este tipo de análise }\end{array}$ & $\begin{array}{c}\text { Apresenta as diretrizes em um } \\
\text { apêndice }\end{array}$ \\
\hline
\end{tabular}


um mínimo de 30 ciclos e Marasteanu et al. (2005) sugerem valores superiores a 100 para materiais com alta elasticidade $\left(\delta<70^{\circ}\right)$.

\subsection{Objetivos da pesquisa}

O presente estudo tem por objetivo principal comparar os resultados dos ensaios de fluência e recuperação sob tensão múltipla (MSCR) segundo os protocolos da ASTM D7405-10a e da AASHTO T350-14, tendo como base a caracterização de ligantes asfálticos modificados com borracha moída (CAP+borracha), ácido polifosfórico (CAP+PPA) e uma combinação dos dois modificadores (CAP+borracha+PPA). Dentre os objetivos secundários da pesquisa, podem-se citar os seguintes:

- Avaliar as variações nos valores de $\mathrm{R}$ e de $\mathrm{J}_{\mathrm{nr}}$ em função do número de ciclos de fluência e recuperação, de modo a observar se ambos os procedimentos permitem a observação do steady state em ligantes asfálticos modificados;

- Verificar a viabilidade da substituição de parte do percentual de borracha moída pelo PPA na modificação do CAP puro, sem que haja prejuízos significativos à sua suscetibilidade ao acúmulo de deformação permanente no ensaio MSCR; e

- Selecionar a melhor formulação dentre as três estudadas na pesquisa com base nos resultados do ensaio $\operatorname{MSCR}\left(\mathbf{J}_{\mathrm{nr}}\right.$ menor, $\mathrm{R}$ maior e $\mathrm{J}_{\mathrm{nr} \text {, diff }}$ menor) e para ambas as normas.

\section{MATERIAIS E ENSAIOS DE LABORATÓRIO}

\subsection{Materiais, preparação das formulações e envelhecimento a curto prazo}

Os seguintes materiais foram selecionados para a preparação das formulações: (i) um CAP 50/70 (PG 64-xx e grau PG contínuo de $67,0^{\circ} \mathrm{C}$ ) oriundo da refinaria ReplanPetrobras de Paulínia (SP); (ii) a borracha moída de pneu procedente da trituração das bandas de rodagem de pneus de veículos de passeio e com $100 \%$ das partículas passantes na peneira \#30 $(0,60 \mathrm{~mm})$; e (iii) o ácido polifosfórico de designação comercial Innovalt ${ }^{\circledR}$ E200, fornecido pela Innophos Inc. dos Estados Unidos. Todas as formulações são classificadas como PG 76-xx na Tabela 3 da norma da AASHTO para o Superpave ${ }^{\circledR}$ (AASHTO, 2009), conforme dados técnicos constantes na Tabela 2. O CAP+PPA foi preparado em um misturador de baixo cisalhamento Fisatom $722 \mathrm{D}$, ao passo que o CAP+borracha e o CAP+borracha+PPA foram preparados em um misturador de alto cisalhamento Silverson L4RT.

O envelhecimento dos ligantes asfálticos a curto prazo seguiu as etapas e procedimentos descritos na norma D2872 da ASTM (ASTM, 2012). Neste ensaio, $35 \mathrm{~g} \pm 0,5$ $\mathrm{g}$ de CAP são despejados em um frasco de vidro padronizado e o conjunto é colocado em uma estufa rotativa a $163^{\circ} \mathrm{C}$ por $85 \mathrm{~min}$. Este processo simula o envelhecimento sofrido pelo CAP durante a mistura com os agregados na usina (usinagem) e a compactação no campo.

\subsection{Ensaios de fluência e recuperação (MSCR) e análise dos dados}

Os procedimentos MSCR (ASTM, 2010; AASHTO, 2014) foram realizados em um reômetro da marca TA Instruments, modelo AR-2000ex. Pelo menos duas réplicas por ligante asfáltico envelhecido no RTFOT foram ensaiadas nas temperaturas de $52,58,64,70$ e $76^{\circ} \mathrm{C}$, tanto utilizando a norma D7405-10a da ASTM quanto a norma T350-14 da AASHTO. Os parâmetros R (percentual de recuperação), $\mathrm{J}_{\mathrm{nr}}$ (compliância não-recuperável) e $\mathrm{J}_{\mathrm{nr}}$, diff (diferença percentual entre as compliâncias, Equação (2)) foram calculados para o CAP puro e para os materiais modificados com PPA, borracha e borracha+PPA e, para fins de análise estatística, os desvios-padrão populacionais $\left(\sigma_{\mathrm{P}}\right)$ e os Coeficientes de Variação (CV) também foram determinados.

$$
J_{n r, \text { diff }}=\frac{J_{n r} 3200-J_{n r} 100}{J_{n r} 100} \times 100
$$

em que: $J_{n r}$, diff: diferença percentual entre as compliâncias, em porcentagem;

$J_{n r} 100$ : compliância não-recuperável a 100 $\mathrm{Pa}\left[\mathrm{Pa}^{-1}, \mathrm{kPa}^{-1}\right] ; \mathrm{e}$

$J_{n r}$ 3200: compliância não-recuperável a 3.200 $\mathrm{Pa}\left[\mathrm{Pa}^{-1}, \mathrm{kPa}^{-1}\right]$.

As comparações entre os resultados do MSCR segundo as duas normas (ASTM, 2010; AASHTO, 2014) foram feitas considerando os seguintes aspectos: (i) os valores numéricos de $\mathrm{R}, \mathrm{J}_{\mathrm{nr}}$ e $\mathrm{J}_{\mathrm{nr} \text {, diff }}$; (ii) as variações entre os valores de $\mathrm{R}$ e $\mathrm{J}_{\mathrm{nr}}$ por ciclo com base em gráficos típicos de comportamento destas variáveis; e (iii) a quantificação destas variações em termos de $\sigma_{\mathrm{P}}$ e CV por temperatura e nível de tensão. O trabalho de Golalipour (2011) serviu de referência para a preparação deste documento, especialmente no que diz respeito à consideração de formulações com o mesmo grau PG em temperaturas altas (76-xx). Entretanto, o presente estudo se destaca principalmente pela comparação direta entre os resultados do MSCR segundo as normas da ASTM e da AASHTO, algo que não foi feito por Golalipour (2011). Publicações mais recentes tendem a utilizar apenas um dos protocolos na avaliação da resistência dos ligantes asfálticos à deformação permanente como, por exemplo, DuBois et al. (2014) e Jafari e Babazadeh (2016) no caso das normas AASHTO TP70-13 (equivalente à AASHTO T350-14) e ASTM D7405-10a, respectivamente.

\section{RESULTADOS E DISCUSSÃO}

\subsection{Parâmetros do ensaio MSCR segundo a ASTM D7405-10a e a AASHTO T350-14}

A Tabela 3 mostra os percentuais de recuperação e as compliâncias não-recuperáveis dos ligantes asfálticos segundo a norma da ASTM.

Tabela 2. Teores de modificadores, variáveis de processamento e graus PG contínuos (true grades) das formulações estudadas

\begin{tabular}{|c|c|c|c|c|c|c|}
\hline Formulação & $\begin{array}{c}\text { Borracha } \\
(\% \text { em massa) } \\
\end{array}$ & $\begin{array}{c}\text { PPA } \\
\text { (\% em massa) } \\
\end{array}$ & $\begin{array}{c}\text { Tempo de } \\
\text { mistura (min) }\end{array}$ & $\begin{array}{c}\text { Temperatura } \\
\left({ }^{\circ} \mathbf{C}\right)\end{array}$ & $\begin{array}{c}\begin{array}{c}\text { Rotação } \\
\text { (rpm) }\end{array} \\
\end{array}$ & True grade $\left({ }^{\circ} \mathrm{C}\right)$ \\
\hline $\mathrm{CAP}+\mathrm{PPA}$ & - & 1,2 & 30 & 130 & 300 & 77,8 \\
\hline CAP+borracha & 14,0 & - & 90 & 190 & 4.000 & 80,2 \\
\hline $\mathrm{CAP}+$ borracha+PPA & 11,0 & 0,5 & $120 *$ & 190 & 4.000 & 80,8 \\
\hline
\end{tabular}

*O ácido polifosfórico foi adicionado à formulação após 90 min de mistura da borracha moída com o CAP. 
A Tabela 4 apresenta estes mesmos parâmetros, porém obtidos segundo a norma da AASHTO. Finalmente, a Tabela 5 destaca os resultados do parâmetro $\mathrm{J}_{\mathrm{nr} \text {, diff para as }}$ formulações selecionadas. Em linhas gerais, pode-se dizer que o protocolo do ensaio MSCR, segundo a AASHTO, fornece resultados melhores (percentuais de 3 a $650 \%$ maiores e compliâncias de 9 a $49 \%$ menores) se comparados aos resultados obtidos utilizando o protocolo da ASTM, sobretudo para o CAP+borracha e o CAP+borracha+PPA. Em outras palavras, a norma da ASTM apresenta estimativas mais conservadoras no que tange à capacidade do ligante asfáltico em resistir à deformação permanente. Entretanto, os cálculos de $\mathrm{J}_{\mathrm{nr}}$, segundo a AASHTO, levaram a incrementos de $\mathrm{J}_{\mathrm{nr}}$, diff para todos os ligantes asfálticos, à exceção do CAP+PPA (temperatura de $76^{\circ} \mathrm{C}$ ) e do CAP 50/70. Isto significa que a resposta nãolinear do material é subestimada no protocolo da ASTM, bem como a suscetibilidade à deformação permanente em situações inesperadas de carregamento e temperatura.

No caso dos percentuais de recuperação, houve um incremento médio de $58 \%$ com a mudança do protocolo de ensaio de ASTM para AASHTO. O material que mais se beneficiou com esta mudança foi o CAP+borracha (incremento médio de $101 \%$ ), seguido pelo CAP+borracha+PPA (média de $40 \%$ ) e, finalmente, pelo CAP+PPA (média de $36 \%$ ). O CAP puro foi uma exceção à regra, pois, dependendo do nível de tensão, foram observadas mais reduções que aumentos nos valores de $\mathrm{R}$ ao migrar do protocolo D7405-10a para o protocolo T350-14. Este foi o caso dos valores obtidos na tensão de $100 \mathrm{~Pa}$, na qual as recuperações diminuíram de um protocolo para outro nas temperaturas de $52^{\circ} \mathrm{C}(8 \%)$ e $64^{\circ} \mathrm{C}(75 \%)$. Já para a tensão de $3.200 \mathrm{~Pa}$, as recuperações do CAP puro seguiram a tendência dos outros materiais e registraram aumentos de 10 e $110 \%$ nas temperaturas de 52 e $58^{\circ} \mathrm{C}$, respectivamente.

Independentemente dos resultados obtidos para o $\mathrm{CAP}$ puro a 52 e $58^{\circ} \mathrm{C}$, há que se considerar que as temperaturas críticas do pavimento sejam da ordem de 64 ou $70^{\circ} \mathrm{C}$, considerando os graus de desempenho atribuídos nos estudos de Leite e Tonial (1994) e Cunha et al. (2007), de modo que, nestas temperaturas, a recuperação elástica do CAP puro é praticamente nula, conforme se constata na Tabela 3. Os resultados nas temperaturas de 64 e $70^{\circ} \mathrm{C}$ seriam indicativos de que, a rigor, não haveria distinção entre os procedimentos no que se refere à medida da recuperação elástica do CAP puro.

Diferentemente dos percentuais de recuperação, as compliâncias não-recuperáveis sofreram reduções para todos os ligantes asfálticos quando da mudança do protocolo da ASTM para o da AASHTO. As reduções foram de $30 \%$ em média para todos os ligantes asfálticos (mínimo de $10 \%$ e máximo de $48 \%$ ), sendo maiores para o CAP+borracha (42\%) e menores para o CAP+borracha+PPA (40\%), o CAP+PPA (27\%) e o CAP 50/70 (12\%). Isto significa que, à luz do procedimento da AASHTO, as formulações possuem uma propensão menor ao acúmulo de deformação permanente. Entretanto, a sensibilidade à tensão e o grau de não-linearidade da resposta reológica $\left(\mathrm{J}_{\mathrm{nr} \text {, diff }}\right)$ se mostram maiores para os ligantes asfálticos modificados no procedi- mento da AASHTO em comparação ao da ASTM (em média, 25\% maiores), sobretudo para o CAP+borracha e o CAP+borracha+PPA.

Ainda sobre os valores do parâmetro $\mathrm{J}_{\mathrm{nr} \text {, diff, cabe res- }}$ saltar que a especificação Superpave ${ }^{\circledR}$ estabelece um limite de $75 \%$ para o seu valor máximo com o intuito de controlar a não-linearidade da resposta do ligante asfáltico e a sua sensibilidade ao acúmulo de deformação em situações nãoprevistas de carregamento e temperatura. Neste aspecto, é possível observar que o CAP+borracha supera este valor máximo nas temperaturas de 64,70 e $76^{\circ} \mathrm{C}$, tanto pelo procedimento da norma D7405-10a quanto pelo procedimento da norma T350-14. Por sua vez, o CAP+borracha+PPA supera o valor de $75 \%$ apenas a $76^{\circ} \mathrm{C}$ pelo procedimento da ASTM e nas temperaturas de 70 e $76^{\circ} \mathrm{C}$ pelo procedimento da AASHTO. Assim, o aumento dos valores de $\mathrm{J}_{\mathrm{nr}}$, diff , observado quando se empregou o procedimento da AASHTO, não foi suficiente para fazer com que muitos ligantes asfálticos ultrapassassem o limite de $75 \%$ em outras temperaturas de ensaio. $\mathrm{O} \mathrm{CAP}+$ borracha+PPA chegou próximo deste limite na temperatura de $64^{\circ} \mathrm{C}(65,2 \%)$ segundo a T350-14, assim como o CAP+borracha a $58^{\circ} \mathrm{C}(72,9 \%)$. Já para o CAP 50/70, reduções de $\mathrm{J}_{\mathrm{nr}}$, diff são observadas em todas as temperaturas após a mudança do protocolo de ensaio, exceção feita à temperatura de $58^{\circ} \mathrm{C}$ (aumento de $10,9 \%$ para $11,1 \%)$.

Em um primeiro momento, pode-se pensar que as reduções das compliâncias não-recuperáveis, obtidas quando da aplicação do protocolo T350-14 da AASHTO, poderiam levar a um incremento dos níveis adequados de tráfego para todos os ligantes asfálticos. Em outras palavras, seria de se supor que os materiais fossem capazes de suportar tráfegos mais pesados quando analisados segundo o protocolo AASHTO. Os dados da Tabela 6 indicam que esta conclusão é parcialmente verdadeira para as formulações e condições de temperatura estudadas. Em termos gerais, nota-se que as reduções de $\mathrm{J}_{\mathrm{nr}}$ a 3.200 Pa não foram suficientes para aumentar o nível de tráfego a que o ligante asfáltico pode ser submetido no pavimento quando as temperaturas são mais baixas (até $58^{\circ} \mathrm{C}$ ). Entretanto, duas das três formulações (CAP+borracha e CAP+borracha+PPA) foram beneficiadas com estas reduções de $\mathrm{J}_{\mathrm{nr}}$ nas temperaturas de $64 \mathrm{e}$ $70^{\circ} \mathrm{C}$ : os tráfegos subiram um nível na escala da especificação Superpave ${ }^{\circledR}$ em ambos os casos, passando de muito pesado (V) para extremamente pesado (E) a $64^{\circ} \mathrm{C}$ e de pesado (H) para muito pesado $(\mathrm{V})$ a $70{ }^{\circ} \mathrm{C}$. O CAP+PPA também foi beneficiado com esta redução, porém apenas na temperatura de $76^{\circ} \mathrm{C}$ (de padrão $(\mathrm{S})$ para pesado $(\mathrm{H})$ ). Por sua vez, o CAP puro não mostrou qualquer alteração nos níveis adequados de tráfego para os casos em que $\mathrm{J}_{\mathrm{nr}} \leq 4,0 \mathrm{kPa}^{-1}$, o que seria esperado, já que as alterações no procedimento de ensaio feitas pela AASHTO visaram corrigir problemas do protocolo observados especificamente quando da obtenção das medidas em ligantes asfálticos modificados.

Pode-se observar que, em termos gerais, o CAP+borracha foi a formulação que mais se beneficiou com a alteração do protocolo do ensaio MSCR (maiores aumentos de R e reduções de $\mathrm{J}_{\mathrm{nr}}$ ) em comparação ao $\mathrm{CAP}+$ borracha+PPA e ao CAP+PPA. 
Tabela 3. Percentuais de recuperação e compliâncias não-recuperáveis para as condições de ensaio segundo a norma ASTM D7405-10a

\begin{tabular}{|c|c|c|c|c|c|c|c|c|c|c|}
\hline \multirow{2}{*}{ Ligante asfáltico } & \multicolumn{5}{|c|}{ Recuperações a $100 \mathrm{~Pa}(\mathrm{R}, \%)$} & \multicolumn{5}{|c|}{ Recuperações a 3.200 Pa $(\mathrm{R}, \%)$} \\
\hline & $52^{\circ} \mathrm{C}$ & $58^{\circ} \mathrm{C}$ & $64^{\circ} \mathrm{C}$ & $7^{\circ} \mathrm{C}$ & $7^{\circ} \mathrm{C}$ & $52^{\circ} \mathrm{C}$ & $\mathbf{5 8}^{\circ} \mathrm{C}$ & $64^{\circ} \mathrm{C}$ & $\mathbf{7 0}^{\circ} \mathrm{C}$ & $7^{\circ} \mathrm{C}$ \\
\hline CAP 50/70 & 12,4 & 5,8 & 1,0 & 0,0 & 0,0 & 8,9 & 0,5 & 0,0 & 0,0 & 0,0 \\
\hline $\mathrm{CAP}+\mathrm{PPA}$ & 63,7 & 55,2 & 44,6 & 34,1 & 24,0 & 62,4 & 49,8 & 31,3 & 12,1 & 0,8 \\
\hline CAP+borracha & 64,0 & 57,9 & 50,1 & 41,1 & 34,7 & 53,2 & 37,4 & 19,8 & 7,5 & 0,8 \\
\hline $\mathrm{CAP}+$ borracha+PPA & 61,0 & 53,0 & 44,5 & 34,0 & 25,5 & 54,0 & 38,1 & 20,0 & 6,6 & 0,0 \\
\hline \multirow{2}{*}{ Ligante asfáltico } & \multicolumn{5}{|c|}{ Compliâncias a $100 \mathrm{~Pa}\left(\mathrm{Jn}_{\mathrm{nr}}, \mathrm{kPa}^{-1}\right)$} & \multicolumn{5}{|c|}{ Compliâncias a 3.200 Pa $\left(\mathrm{Jnn}_{\mathrm{nr}}, \mathrm{kPa}^{-1}\right)$} \\
\hline & $52^{\circ} \mathrm{C}$ & $\mathbf{5 8}^{\circ} \mathrm{C}$ & $64^{\circ} \mathrm{C}$ & $\mathbf{7 0}^{\circ} \mathrm{C}$ & $7^{\circ} \mathrm{C}$ & $52^{\circ} \mathbf{C}$ & $\mathbf{5 8}^{\circ} \mathrm{C}$ & $64^{\circ} \mathrm{C}$ & $\mathbf{7 0}^{\circ} \mathrm{C}$ & $\mathbf{7 6}^{\circ} \mathrm{C}$ \\
\hline CAP 50/70 & 0,33 & 0,92 & 2,44 & 5,96 & 13,53 & 0,35 & 1,02 & 2,78 & 6,79 & 15,23 \\
\hline $\mathrm{CAP}+\mathrm{PPA}$ & 0,03 & 0,08 & 0,23 & 0,59 & 1,48 & 0,03 & 0,09 & 0,29 & 0,86 & 2,38 \\
\hline CAP+borracha & 0,05 & 0,13 & 0,32 & 0,78 & 1,65 & 0,07 & 0,20 & 0,59 & 1,48 & 3,38 \\
\hline $\mathrm{CAP}+$ borracha+PPA & 0,05 & 0,13 & 0,33 & 0,81 & 1,83 & 0,06 & 0,18 & 0,53 & 1,39 & 3,30 \\
\hline
\end{tabular}

Tabela 4. Percentuais de recuperação e compliâncias não-recuperáveis para as condições de ensaio segundo a norma AASHTO T350-14

\begin{tabular}{|c|c|c|c|c|c|c|c|c|c|c|}
\hline \multirow{2}{*}{ Ligante asfáltico } & \multicolumn{5}{|c|}{ Recuperações a $100 \mathrm{~Pa}(\mathrm{R}, \%)$} & \multicolumn{5}{|c|}{ Recuperações a 3.200 Pa (R, \%) } \\
\hline & $52^{\circ} \mathrm{C}$ & $58^{\circ} \mathrm{C}$ & $64^{\circ} \mathrm{C}$ & $70^{\circ} \mathrm{C}$ & $76^{\circ} \mathrm{C}$ & $52^{\circ} \mathrm{C}$ & $\mathbf{5 8}^{\circ} \mathrm{C}$ & $64^{\circ} \mathrm{C}$ & $7^{\circ} \mathrm{C}$ & $76^{\circ} \mathrm{C}$ \\
\hline CAP 50/70 & 11,4 & 6,3 & 0,3 & 0,0 & 0,0 & 9,8 & 1,1 & 0,0 & 0,0 & 0,0 \\
\hline $\mathrm{CAP}+\mathrm{PPA}$ & 69,6 & 60,7 & 50,0 & 38,8 & 25,5 & 64,8 & 54,1 & 37,2 & 17,1 & 2,7 \\
\hline CAP+borracha & 75,0 & 69,9 & 64,5 & 56,4 & 44,5 & 62,8 & 50,1 & 32,9 & 16,5 & 6,0 \\
\hline $\mathrm{CAP}+$ borracha+PPA & 71,4 & 64,6 & 55,4 & 45,8 & 33,2 & 62,8 & 49,7 & 32,2 & 14,8 & 4,0 \\
\hline \multirow{2}{*}{ Ligante asfáltico } & \multicolumn{5}{|c|}{ Compliâncias a 100 Pa $\left(\mathrm{Jn}_{\mathrm{nr}}, \mathrm{kPa}^{-1}\right)$} & \multicolumn{5}{|c|}{ Compliâncias a 3.200 Pa $\left(\mathrm{Jn}_{\mathrm{nr}}, \mathrm{kPa}^{-1}\right)$} \\
\hline & $52^{\circ} \mathbf{C}$ & $58^{\circ} \mathrm{C}$ & $64^{\circ} \mathrm{C}$ & $7^{\circ}{ }^{\circ} \mathrm{C}$ & $76^{\circ} \mathrm{C}$ & $52^{\circ} \mathrm{C}$ & $58^{\circ} \mathrm{C}$ & $64^{\circ} \mathrm{C}$ & $7^{\circ} \mathrm{C}$ & $76^{\circ} \mathrm{C}$ \\
\hline CAP 50/70 & 0,30 & 0,82 & 2,19 & 5,28 & 11,78 & 0,31 & 0,91 & 2,39 & 5,85 & 13,11 \\
\hline $\mathrm{CAP}+\mathrm{PPA}$ & 0,02 & 0,06 & 0,16 & 0,43 & 1,15 & 0,02 & 0,07 & 0,21 & 0,63 & 1,83 \\
\hline CAP+borracha & 0,03 & 0,07 & 0,17 & 0,40 & 1,00 & 0,04 & 0,12 & 0,35 & 0,93 & 2,21 \\
\hline $\mathrm{CAP}+$ borracha+PPA & 0,03 & 0,07 & 0,19 & 0,47 & 1,15 & 0,04 & 0,11 & 0,31 & 0,86 & 2,16 \\
\hline
\end{tabular}

Tabela 5. Diferenças percentuais entre as compliâncias não-recuperáveis (parâmetro $J_{\mathrm{nr}}$ difit) segundo as normas da ASTM e da AASHTO

\begin{tabular}{ccccccccccc}
\hline \multirow{2}{*}{ Ligante asfáltico } & \multicolumn{4}{c}{$\mathbf{J}_{\mathbf{n r} \text {, diff }}(\mathbf{\%})$, ASTM D7405-10a } & \multicolumn{4}{c}{$\mathbf{J}_{\mathbf{n r}}$, diff $(\boldsymbol{\%}), \mathbf{A A S H T O} \mathbf{T 3 5 0 - 1 4}$} \\
\cline { 2 - 11 } & $\mathbf{5 2}^{\circ} \mathbf{C}$ & $\mathbf{5 8}^{\circ} \mathbf{C}$ & $\mathbf{6 4}^{\circ} \mathbf{C}$ & $\mathbf{7 0}^{\circ} \mathbf{C}$ & $\mathbf{7 6}^{\circ} \mathbf{C}$ & $\mathbf{5 2}^{\circ} \mathbf{C}$ & $\mathbf{5 8}^{\circ} \mathbf{C}$ & $\mathbf{6 4}^{\circ} \mathbf{C}$ & $\mathbf{7 0}^{\circ} \mathbf{C}$ & $\mathbf{7 6}^{\circ} \mathbf{C}$ \\
\hline CAP 50/70 & 6,1 & 10,9 & 14,0 & 14,0 & 12,6 & 3,2 & 11,1 & 9,4 & 10,8 & 11,3 \\
CAP+PPA & 0,0 & 12,5 & 26,7 & 46,2 & 61,4 & 17,5 & 16,5 & 26,9 & 46,5 & 58,6 \\
CAP+borracha & 33,6 & 56,5 & 81,5 & 90,3 & 104,8 & 53,6 & 72,9 & 109,3 & 130,9 & 120,5 \\
CAP+borracha+PPA & 20,0 & 38,5 & 61,5 & 72,0 & 80,1 & 32,1 & 46,2 & 65,2 & 84,8 & 87,6 \\
\hline
\end{tabular}

Tabela 6. Níveis adequados de tráfego segundo as normas da ASTM e da AASHTO

\begin{tabular}{ccccccccccc}
\hline \multirow{2}{*}{ Ligante asfáltico } & \multicolumn{3}{c}{ Níveis de tráfego, ASTM D7405-10a* } & \multicolumn{4}{c}{ Níveis de tráfego, AASHTO T350-14* } \\
\cline { 2 - 10 } & $\mathbf{5 2}^{\circ} \mathbf{C}$ & $\mathbf{5 8}^{\circ} \mathbf{C}$ & $\mathbf{6 4}^{\circ} \mathbf{C}$ & $\mathbf{7 0}^{\circ} \mathbf{C}$ & $\mathbf{7 6}^{\circ} \mathbf{C}$ & $\mathbf{5 2}^{\circ} \mathbf{C}$ & $\mathbf{5 8}^{\circ} \mathbf{C}$ & $\mathbf{6 4}^{\circ} \mathbf{C}$ & $\mathbf{7 0}^{\circ} \mathbf{C}$ & $\mathbf{7 6}^{\circ} \mathbf{C}$ \\
\hline CAP 50/70 & E & V & S & - & - & E & V & S & - & - \\
CAP+PPA & E & E & E & V & S & E & E & E & V & H \\
CAP+borracha & E & E & V & H & S & E & E & E & V & S \\
CAP+borracha+PPA & E & E & V & H & S & E & E & E & V & S
\end{tabular}

$*$ E = tráfego extremamente pesado $\left(\mathrm{J}_{\mathrm{nr}} \leq 0,5 \mathrm{kPa}^{-1}\right) ; \mathrm{V}=$ tráfego muito pesado $\left(0,5 \mathrm{kPa}^{-1}<\mathrm{J}_{\mathrm{nr}} \leq 1,0 \mathrm{kPa}^{-1}\right) ; \mathrm{H}=$ tráfego pesado $\left(1,0 \mathrm{kPa}^{-1}<\mathrm{J}_{\mathrm{nr}} \leq 2,0\right.$ $\left.\mathrm{kPa}^{-1}\right) ; \mathrm{S}=$ tráfego padrão $\left(2,0 \mathrm{kPa}^{-1}<\mathrm{J}_{\mathrm{nr}} \leq 4,0 \mathrm{kPa}^{-1}\right)$.

Por outro lado, esta mesma formulação também apresenta uma sensibilidade excessiva à tensão $\left(\mathrm{J}_{\mathrm{nr} \text {, diff }}>75 \%\right)$ em diversas temperaturas de ensaio $\left(64,70\right.$ e $\left.76^{\circ} \mathrm{C}\right)$, tanto na norma D7405 da ASTM quanto na norma T350 da AASHTO. Em outras palavras, o uso do CAP+borracha em pavimentos rodoviários deve ser precedido de uma avaliação criteriosa dos níveis de tensão reais aplicados pelos veículos, pois condições atípicas de carregamento podem levar ao acúmulo de deformações permanentes excessivas no revestimento asfáltico. Finalmente, a substituição de parte do teor de borracha moída pelo PPA na formulação $\mathrm{CAP}+$ borracha+PPA propiciou uma redução considerável das sensibilidades à tensão, muito embora os resultados do MSCR sejam um pouco inferiores aos do CAP+borracha para uma mesma temperatura de ensaio (até 33\% de redução no parâmetro R e até $18 \%$ de aumento no parâmetro $\mathrm{J}_{\mathrm{nr}}$ ).

\subsection{Análise estatística das variações dos parâmetros do MSCR por ciclo}

Como já destacado, um dos motivos apontados pelos pesquisadores para aumentar o número de ciclos de fluência e recuperação no RCRT e no MSCR foi a obtenção de resultados mais próximos ao estado de steady state (Bahia et al., 2001; Marasteanu et al., 2005; Delgadillo et al., 2006b; Golalipour, 2011). Neste estado, os efeitos da elasticidade retardada são reduzidos e o acúmulo de deformação no material segue uma taxa de crescimento aproximadamente constante. Embora não haja um consenso definitivo na literatura acerca do número de ciclos necessários para que o 
steady state seja claramente observado nos ligantes asfálticos modificados, a norma AASHTO T350-14 adotou um valor fixo de 20 ciclos a $100 \mathrm{~Pa}$ e não alterou o total de ciclos a 3.200 Pa (10 ciclos). Entretanto, o método de cálculo dos parâmetros $\mathrm{R}$ e $\mathrm{J}_{\mathrm{nr}}$ passou a desconsiderar os primeiros ciclos de fluência e recuperação a $100 \mathrm{~Pa}$ e leva em consideração apenas os últimos dez ciclos (do $11^{\circ}$ ao $20^{\circ}$ ). É provável que estes ciclos iniciais do MSCR tenham sido desconsiderados nas versões atualizadas da norma da AASHTO devido ao alto grau de variabilidade dos resultados individuais dos parâmetros $\mathrm{R}$ e $\mathrm{J}_{\mathrm{nr}}$ (Golalipour, 2011).

As Figuras 1 e 2 ilustram as variações dos resultados de $\mathrm{R}$ e $\mathrm{J}_{\mathrm{nr}}$ por ciclo de fluência e recuperação a 64 e $70^{\circ} \mathrm{C}$, respectivamente, e segundo o protocolo da AASHTO. Por brevidade, apenas os resultados obtidos por meio deste protocolo são exibidos no presente trabalho. Os dados do CAP 50/70 (PG 64-xx) não foram plotados porque o efeito da elasticidade retardada na resposta reológica deste material não é tão significativo quanto no caso dos ligantes asfálticos modificados.

Observa-se que os valores das recuperações e das compliâncias não-recuperáveis tendem a estabilizar depois de um certo número de ciclos a $100 \mathrm{~Pa}$. Isto indica que os resultados de $\mathrm{R}$ são subestimados e os de $\mathrm{J}_{\text {nr }}$ são superestimados nos primeiros ciclos de creep-recovery. Nota-se também que, na tensão de $3.200 \mathrm{~Pa}$, um equilíbrio dos resultados do MSCR já pode ser constatado a partir do $5^{\circ}$ e $6^{\circ}$ ciclos ( $\mathrm{R}$ e $\mathrm{J}_{\mathrm{nr}}$, respectivamente). As três formulações com PPA, borracha e borracha+PPA se aproximam do steady state nos ciclos finais a $100 \mathrm{~Pa}$, especialmente após o $15^{\circ}$ ciclo. Comportamentos parecidos foram visualizados para os outros ensaios MSCR.

É importante ressaltar que a subestimativa dos percentuais de recuperação e a superestimativa das compliâncias não-recuperáveis nos ciclos iniciais do MSCR (ver os

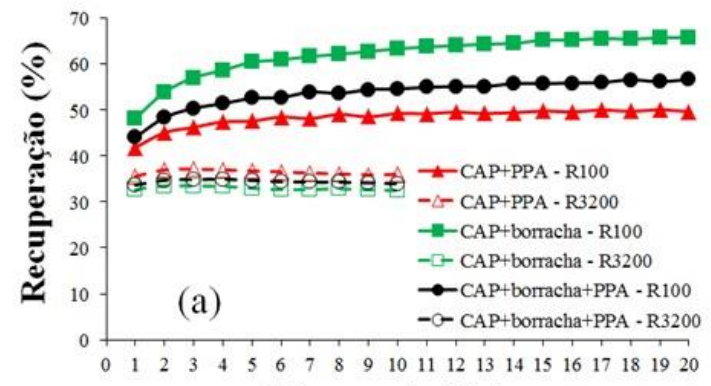

Número do Ciclo primeiros e últimos valores nas Figuras 1 e 2) podem acarretar despesas desnecessárias na escolha da formulação mais adequada para uma situação específica de temperatura e nível de tráfego. Por exemplo, a observância do protocolo da norma ASTM D7405-10a conduz a resultados indicativos de que o CAP+borracha e o CAP+borracha+PPA não suportariam um tráfego extremamente pesado (E) na temperatura de $64^{\circ} \mathrm{C}$ (Tabela 6), que é típica dos pavimentos brasileiros (Leite e Tonial, 1994; Cunha et al., 2007). Por outro lado, o protocolo da norma AASHTO T350-14 proporciona resultados que indicam que ambos os materiais poderiam suportar um tráfego de nível "E" a $64^{\circ} \mathrm{C}$. Em outras palavras, a empresa que avalia seus produtos à luz do protocolo da norma D7405-10a da ASTM teria que produzir outra formulação com mais borracha moída, de modo a alcançar o tráfego nível "E" a $64^{\circ} \mathrm{C}$. Tal prática aumentaria não só o custo do produto, por conta da adição de concentrações mais altas de borracha moída e de aditivos, mas também os custos da produção da camada asfáltica. Estes últimos aumentariam por conta, por exemplo, do aumento do consumo de combustível para aumentar as temperaturas na usina e do aumento do esforço de compactação da camada. Já a empresa que avalia seus produtos à luz do protocolo da norma T350-14 da AASHTO poderia se beneficiar, na extensão em que poderia fornecer um produto mais barato e com melhor trabalhabilidade.

A Tabela 7 quantifica as variações entre os resultados de $\mathrm{R}$ e $\mathrm{J}_{\mathrm{nr}}$ por ciclo de fluência e recuperação a $64^{\circ} \mathrm{C}$, considerando os desvios-padrão populacionais e os respectivos coeficientes de variação com todos os ciclos e apenas nos últimos cinco ciclos a 100 e a $3.200 \mathrm{~Pa}$ (ASTM D7405-10a), ou ainda apenas nos últimos 10 ciclos a $100 \mathrm{~Pa}$ (AASHTO T350-14).

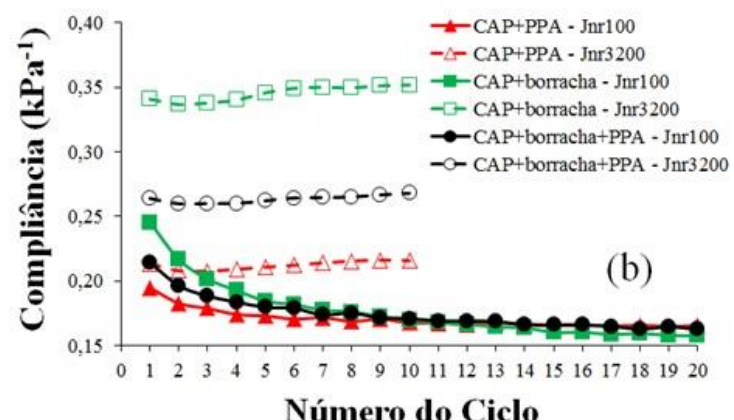

Figura 1. Variações nos valores dos percentuais de recuperação $R(a)$ e compliâncias não-recuperáveis $J_{n r}$ (b) por ciclo a $64^{\circ} \mathrm{C}$ e nas tensões de 100 e $3.200 \mathrm{~Pa}$

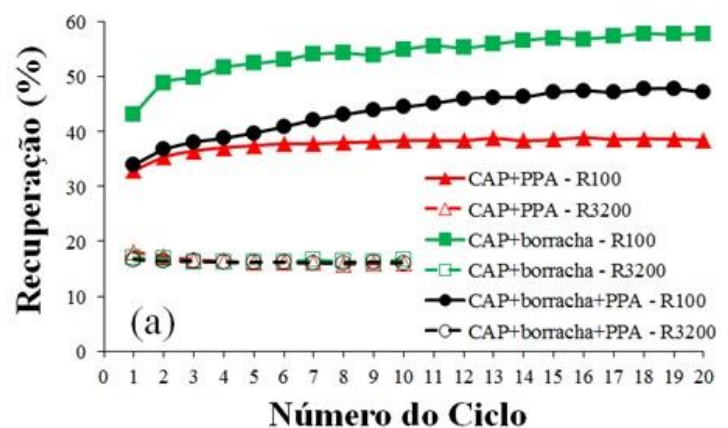

Figura 2. Variações nos valores dos percentuais de recuperação $R$ (a) e das compliâncias não-recuperáveis $J_{n r}(b)$ por ciclo a $70^{\circ} \mathrm{C}$ e nas tensões de 100 e $3.200 \mathrm{~Pa}$ 
Ensaios MSCR Segundo as Normas ASTM D7405-10a e AASHTO T350-14: Um Estudo de Caso Envolvendo Ligantes Asfálticos Modificados

Tabela 7. Desvios-padrão, coeficientes de variação e valores médios de $\mathrm{R}$ (percentual de recuperação) e $\mathrm{J}_{\text {nr }}$ (compliância não-recuperável) a $64^{\circ} \mathrm{C}$ obtidos segundo as normas ASTM D7405-10a e

\begin{tabular}{|c|c|c|c|c|c|c|}
\hline Norma & Parâmetro* & Descrição do item & CAP 50/70* & CAP+PPA* & CAP+borracha* & CAP+borracha+PPA* \\
\hline \multirow{6}{*}{$\begin{array}{l}\text { D7405-10a (todos os } 10 \text { ciclos e } \\
\text { os últimos cinco a } 100 \mathrm{~Pa} \text { ) }\end{array}$} & \multirow{3}{*}{ R100 } & Valor médio (\%) & $1,0(1,2)$ & $44,4(45,8)$ & $47,8(50,7)$ & $44,8(46,8)$ \\
\hline & & Desvio-padrão $(\sigma \mathrm{P})$, em \% & $0,28(0,08)$ & $1,93(0,25)$ & $3,91(0,87)$ & $2,68(0,52)$ \\
\hline & & Coeficiente de variação (CV), em \% & $27,95(6,07)$ & $4,35(0,54)$ & $8,19(1,71)$ & $5,98(1,12)$ \\
\hline & \multirow{3}{*}{$\mathrm{J}_{\mathrm{nr}} 100$} & Valor médio $\left(\mathrm{kPa}^{-1}\right)$ & $2,436(2,430)$ & $0,227(0,222)$ & $0,364(0,341)$ & $0,325(0,312)$ \\
\hline & & Desvio-padrão $(\sigma \mathrm{P}), \mathrm{em} \mathrm{kPa}^{-1}$ & $0,0072(0,0031)$ & $0,0085(0,0010)$ & $0,0293(0,0065)$ & $0,0175(0,0034)$ \\
\hline & & Coeficiente de variação (CV), em \% & $0,30(0,13)$ & $3,76(0,43)$ & $8,07(1,89)$ & $5,38(1,10)$ \\
\hline \multirow{6}{*}{$\begin{array}{c}\text { T350-14 } \\
\text { (todos os } 20 \text { ciclos e os últimos } \\
\text { dez a } 100 \mathrm{~Pa} \text { ) }\end{array}$} & \multirow{3}{*}{ R100 } & Valor médio (\%) & $1,1(0,5)$ & $48,4(49,7)$ & $62,0(65,0)$ & $53,7(55,8)$ \\
\hline & & Desvio-padrão ( $\sigma \mathrm{P})$, em \% & $1,02(1,02)$ & $1,97(0,27)$ & $4,42(0,71)$ & $3,07(0,56)$ \\
\hline & & Coeficiente de variação $(\mathrm{CV})$, em \% & $88,96(200,54)$ & $4,06(0,36)$ & $7,14(1,09)$ & $5,71(1,00)$ \\
\hline & \multirow{3}{*}{$\mathrm{J}_{\mathrm{nr}} 100$} & Valor médio $\left(\mathrm{kPa}^{-1}\right)$ & $2,131(2,148)$ & $0,171(0,166)$ & $0,177(0,162)$ & $0,175(0,166)$ \\
\hline & & Desvio-padrão $\left(\sigma_{\mathrm{P}}\right), \mathrm{em} \mathrm{kPa}^{-1}$ & $0,0272(0,0276)$ & $0,0072(0,0010)$ & $0,0221(0,0035)$ & $0,0126(0,0023)$ \\
\hline & & Coeficiente de variação (CV), em \% & $1,28(1,29)$ & $4,24(0,59)$ & $12,50(2,15)$ & $7,23(1,38)$ \\
\hline \multirow{6}{*}{$\begin{array}{c}\text { D7405-10a } \\
\text { (todos os } 10 \text { ciclos e os últimos } \\
\text { cinco a } 3.200 \mathrm{~Pa} \text { ) }\end{array}$} & \multirow{3}{*}{ R3200 } & Valor médio (\%) & $0,00(0,00)$ & $31,1(30,7)$ & $17,0(17,0)$ & $20,1(19,9)$ \\
\hline & & Desvio-padrão $(\sigma \mathrm{P})$, em \% & - & $0,44(0,13)$ & $0,22(0,10)$ & $0,24(0,15)$ \\
\hline & & Coeficiente de variação $(\mathrm{CV})$, em \% & - & $1,41(0,42)$ & $1,30(0,57)$ & $1,20(0,75)$ \\
\hline & \multirow{3}{*}{$\mathrm{J}_{\mathrm{nr}} 3200$} & Valor médio $\left(\mathrm{kPa}^{-1}\right)$ & $2,777(2,795)$ & $0,285(0,288)$ & $0,656(0,660)$ & $0,521(0,526)$ \\
\hline & & Desvio-padrão $\left(\sigma_{P}\right), \mathrm{em} \mathrm{kPa}^{-1}$ & $0,0217(0,0085)$ & $0,0036(0,0012)$ & $0,0062(0,0011)$ & $0,0061(0,0022)$ \\
\hline & & Coeficiente de variação (CV), em \% & $0,78(0,31)$ & $1,27(0,40)$ & $0,94(0,17)$ & $1,16(0,42)$ \\
\hline \multirow{6}{*}{$\begin{array}{c}\text { T350-14 } \\
\text { (todos os } 10 \text { ciclos à tensão de } \\
3.200 \mathrm{~Pa} \text { ) }\end{array}$} & \multirow{3}{*}{ R3200 } & Valor médio (\%) & 0,00 & 36,4 & 33,0 & 34,4 \\
\hline & & Desvio-padrão $(\sigma \mathrm{P})$, em \% & - & 0,51 & 0,36 & 0,39 \\
\hline & & Coeficiente de variação (CV), em \% & - & 1,40 & 1,09 & 1,12 \\
\hline & \multirow{3}{*}{$\mathrm{J}_{\mathrm{nr}} 3200$} & Valor médio $\left(\mathrm{kPa}^{-1}\right)$ & 2,388 & 0,212 & 0,345 & 0,263 \\
\hline & & Desvio-padrão $(\sigma \mathrm{P}), \mathrm{em} \mathrm{kPa}^{-1}$ & 0,0190 & 0,0031 & 0,0056 & 0,0027 \\
\hline & & Coeficiente de variação (CV), em \% & 0,80 & 1,46 & 1,61 & 1,03 \\
\hline
\end{tabular}

*Os números entre parênteses se referem aos últimos cinco ou dez ciclos, dependendo da norma e os números fora dos parênteses se referem a todos os ciclos. 
Tal como já visualizado nas Figuras 1 e 2, a desconsideração dos primeiros ciclos nos cálculos de $\mathrm{R}$ e $\mathrm{J}_{\mathrm{nr}}$ acarretou reduções expressivas de $\sigma_{\mathrm{P}}$ e $\mathrm{CV}$ para todos os materiais estudados, mesmo quando se seguiu o protocolo da norma da ASTM. Os valores finais obtidos a $100 \mathrm{~Pa}$ também sofreram as maiores variações de um método de cálculo para outro, especialmente no caso do CAP+borracha e do CAP+borracha+PPA. Interessante observar que o período de condicionamento do CAP no MSCR, caracterizado pela norma T350-14 como sendo os primeiros 10 ciclos a

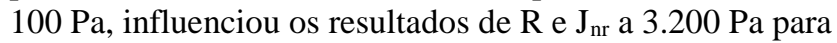
todos os materiais: os percentuais de recuperação e as compliâncias não-recuperáveis podem ser até $95 \%$ maiores e $50 \%$ menores, respectivamente, quando se substitui o protocolo da ASTM pelo da AASHTO. Cabe ressaltar que discussões anteriores sobre o fenômeno de steady state e publicadas na literatura (Bahia et al., 2001; Delgadillo et al., 2006b; Marasteanu et al., 2005) utilizaram tensões baixas de 25 a $300 \mathrm{~Pa}$ - nos ensaios de fluência e recuperação. Com base nos resultados preliminares apresentados nesta pesquisa, acredita-se ser provável que a desconsideração de tensões maiores em muitos estudos sobre o steady state seja decorrente das menores variações de $\mathrm{R}$ e $\mathrm{J}_{\mathrm{nr}}$ nos ciclos iniciais de fluência e recuperação em comparação às tensões menores (e. g., $100 \mathrm{~Pa}$ ).

\section{CONCLUSÕES}

O presente estudo foi dedicado à comparação dos resultados obtidos do ensaio MSCR quando conduzido conforme os procedimentos das normas ASTM D7405-10a e da AASHTO T350-14, que se distinguem, basicamente, pelo número de ciclos de fluência e recuperação e pelo montante de ciclos considerado nos cálculos. As principais conclusões do trabalho são:

- O descarte dos primeiros ciclos de fluência e recuperação nos cálculos de $\mathrm{R}$ e $\mathrm{J}_{\mathrm{nr}}$, conforme recomendado pela norma da AASHTO, proporcionou reduções expressivas na variabilidade dos resultados, mesmo quando se seguiu o protocolo da norma da ASTM; os valores de $\mathrm{R}$ e $\mathrm{J}_{\mathrm{nr}}$ obtidos a $100 \mathrm{~Pa}$ foram os que sofreram as maiores variações de um método de cálculo para outro; observou-se também que o período de condicionamento constante na norma T350-14, de 10 ciclos a $100 \mathrm{~Pa}$, foi capaz de influenciar os resultados de $\mathrm{R}$ e $\mathrm{J}_{\mathrm{nr}}$ a 3.200 Pa: os percentuais de recuperação podem ser até $95 \%$ maiores e as compliâncias nãorecuperáveis até $50 \%$ menores quando se substitui o protocolo da ASTM pelo da AASHTO;

- A migração do protocolo da ASTM para o da AASHTO foi benéfica aos ligantes asfálticos modificados, na extensão em que proporcionou aumento dos valores de $\mathrm{R}$ e redução dos valores de $\mathrm{J}_{\mathrm{nr}}$; em outras palavras, os procedimentos descritos na norma ASTM subestimam as recuperações elásticas dos ligantes asfálticos modificados e superestimam as suas compliâncias não-recuperáveis, em comparação aos procedimentos atuais da norma AASHTO;

- Observaram-se diferenças percentuais entre as compliâncias não-recuperáveis $\left(\mathrm{J}_{\mathrm{nr} \text {, diff }}\right)$ maiores quando foi seguido o procedimento da AASHTO, no entanto, as temperaturas em que o CAP+borracha e o CAP+borracha+PPA se mostram sensíveis a tensão $\left(\mathrm{J}_{\mathrm{nr} \text {, diff }}>75 \%\right)$ são as mesmas nos dois casos: 64,70 e $76^{\circ} \mathrm{C}$, no caso do CAP+borracha, e 70 e $76^{\circ} \mathrm{C}$, no caso do CAP+borracha+PPA; a condução do ensaio por um ou outro procedimento pode levar à atribuição de diferentes níveis de tráfego às misturas asfálticas preparadas com os ligantes asfálticos em questão; por exemplo, o CAP+borracha e o CAP+borracha+PPA suportariam apenas um tráfego muito pesado (classe $\mathrm{V})$, na temperatura de $64^{\circ} \mathrm{C}$, se a avaliação fosse feita segundo o protocolo da ASTM, ao passo que poderiam suportar um tráfego extremamente pesado (E) se a avaliação fosse feita segundo o protocolo da AASHTO; tais resultados conflitantes poderiam obrigar o produtor do ligante asfáltico modificado a elevar o nível de modificação, de modo a alcançar a classe de tráfego exigida para uma dada aplicação, com consequente aumento de custos de produção do material e de construção da camada asfáltica;

- As modificações do CAP com ácido polifosfórico (PPA) ou borracha moída de pneus ou uma combinação de ambos (borracha+PPA) levaram a incrementos no percentual de recuperação $(\mathrm{R})$ e reduções na compliância não-recuperável $\left(\mathrm{J}_{\mathrm{nr}}\right)$ das formulações, em temperaturas oscilando entre 52 e $76{ }^{\circ} \mathrm{C}$, em ambos os protocolos de ensaio; tais benefícios provocados pelos modificadores podem ser traduzidos em uma resposta elástica maior e uma suscetibilidade menor ao acúmulo de deformação permanente;

- Em linhas gerais, o CAP+PPA pode ser considerado como a melhor formulação dentre os ligantes asfálticos modificados estudados, em virtude dos valores mais altos de recuperação elástica e mais baixos de compliância não-recuperável, além da baixa sensibilidade ao incremento do nível de tensão $\left(\mathrm{J}_{\mathrm{nr}}\right.$, diff $<$ $75 \%)$; e

- Apesar da maior sensibilidade à tensão ( $\mathrm{J}_{\mathrm{nr}}$, diff maior $)$, o CAP+borracha possui resultados um pouco melhores ( $\mathrm{R}$ maior e $\mathrm{J}_{\mathrm{nr}}$, menor) e níveis de tráfego semelhantes aos do CAP+borracha+PPA à luz dos protocolos de ensaio das duas normas; isto significa que a substituição de parte do teor de borracha moída pelo PPA não se mostrou benéfica, na ótica da resistência à deformação permanente, a ponto de justificar a adoção de uma formulação com menos borracha moída e um pequeno percentual de ácido polifosfórico.

\section{AGRADECIMENTOS}

O primeiro autor agradece ao Conselho Nacional de Desenvolvimento Científico e Tecnológico (CNPq) pela concessão de uma bolsa de estudo na modalidade doutorado (Processo No. 141377/2012-7). O segundo autor agradece à Fundação de Amparo à Pesquisa do Estado de São Paulo (FAPESP) pela concessão de fundos de auxílio à pesquisa, categoria Jovem Pesquisador (Processo No. 2006/55835-6). 


\section{REFERÊNCIAS}

AASHTO (2009) AASHTO M320 - Standard Specification for Performance-Graded Asphalt Binder. American Association of State Highway and Transportation Officials, Washington, DC.

AASHTO (2014) AASHTO T350 - Standard Method of Test for Multiple Stress Creep Recovery (MSCR) Test of Asphalt Binder Using a Dynamic Shear Rheometer (DSR). American Association of State Highway and Transportation Officials, Washington, DC.

AASHTO (2013) AASHTO TP70 - Standard Method of Test for Multiple Stress Creep Recovery (MSCR) Test of Asphalt Binder Using a Dynamic Shear Rheometer (DSR). American Association of State Highway and Transportation Officials, Washington, DC.

Anderson, M. e J. Bukowski (2012) Using the Multiple-StressCreep-Recovery (MSCR) Test. North Central Asphalt User Producer Group Meeting, Indianapolis, IN. Apresentação em PowerPoint. Disponível em:

$<$ https://engineering.purdue.edu/ ncaupg/Activities/2012/present ation\%202012/Anderson\%20-\%20MSCR\%20AI\%20NCAUPG \%202012.pdf>. Acesso em: 08 jul. 2015.

Apeagyei, A. K. (2014) Flow Number Predictive Models from Volumetric and Binder Properties. Construction and Building Materials, v. 64, p. 240-245.

DOI: 10.1016/j.conbuildmat.2014.04.069.

ASTM (2012) ASTM D2872 - Standard Test Method for Effect of Heat and Air on a Moving Film of Asphalt (Rolling Thin-Film Oven Test). American Society for Testing and Materials, West Conshohocken. DOI: 10.1520/D2872-12E01.

ASTM (2010) ASTM D7405 - Standard Test Method for Multiple Stress Creep and Recovery (MSCR) of Asphalt Binder Using a Dynamic Shear Rheometer. American Society for Testing and Materials, West Conshohocken. DOI: $10.1520 / \mathrm{D} 7405-10 \mathrm{~A}$.

Bahia, H. U.; D. I. Hanson; M. Zeng; H. Zhai; M. A. Khatri e R. M. Anderson (2001) Characterization of Modified Asphalt Binders in Superpave Mix Design. NCHRP Report 459, Transportation Research Board, Washington, DC.

Borges, R. L. (2014) Deformação Permanente em Misturas Asfálticas a Partir do Shift Model Viscoplástico e Ensaios Triaxiais de Carga Repetida. Dissertação (Mestrado em Engenharia de Transportes) - Departamento de Engenharia de Transportes, Universidade Federal do Ceará, Fortaleza.

Cunha, M. B.; J. E. Zegarra e J. L. Fernandes Jr (2007) Revisão da Seleção do Grau de Desempenho (PG) de Ligantes Asfálticos por Estado no Brasil. Anais do $21^{\circ}$ Congresso Nacional de Pesquisa e Ensino em Transportes, ANPET, Rio de Janeiro.

D’Angelo, J.; R. Kluttz; R. Dongré; K. Stephens e L. Zanzotto (2007) Revision of the Superpave High Temperature Binder Specification: The Multiple Stress Creep Recovery Test. Journal of the Association of Asphalt Paving Technologists, v. 76, p. 123-162.

Delgadillo, R.; D. W. Cho e H. Bahia (2006a) Nonlinearity of Repeated Creep and Recovery Binder Test and Relationship with Mixture Permanent Deformation. Transportation Research Record, n. 1962, p. 3-11. DOI: 10.3141/1962-01.

Delgadillo, R.; K. Nam e H. Bahia (2006b) Why do We Need to Change $\mathrm{G}^{*} / \sin \delta$ and How? Road Materials and Pavement
Design, v. 7, n. 1, p. 7-27.

DOI: $10.1080 / 14680629.2006 .9690024$.

DuBois, E.; Y. Mehta e A. Nolan (2014) Correlation Between Multiple Stress Creep Recovery (MSCR) Results and Polymer Modification of Binder. Construction and Building Materials, v. 65, p. 184-190. DOI: 10.1016/j.conbuildmat.2014.04.111.

FHWA (2011) The Multiple Stress Creep Recovery (MSCR) Procedure. Technical Brief, Document No. FHWA-HIF-11-038, Federal Highway Administration, McLean, VA. Disponível em: <http://www.fhwa.dot.gov/pavement/materials/pubs/hif11038/hif 11038.pdf $>$. Acesso em: 08 jul. 2015.

Gibson, N.; X. Qi; A. Shenoy; G. Al-Khateeb; M. E. Kutay; A. Andriescu; K. Stuart; J. Youtcheff e T. Harman (2012) Performance Testing for Superpave and Structural Validation. Final Report No. FHWA-HRT-11-45, Federal Highway Administration, McLean, VA.

Golalipour, A. (2011) Modification of Multiple Stress Creep and Recovery Test Procedure and Usage in Specification. Thesis (Master of Science) - Department of Civil and Environmental Engineering, University of Wisconsin-Madison, Madison.

Jafari, M. e A. Babazadeh (2016) Evaluation of Polyphosphoric Acid-Modified Binders Using Multiple Stress Creep and Recovery and Linear Amplitude Sweep Tests. Road Materials and Pavement Design, v. 17, n. 4, p. 859-876.

DOI: $10.1080 / 14680629.2015 .1132631$.

Kim, S.; G. A. Sholar; T. Byron e J. Kim (2009) Performance of Polymer-Modified Asphalt Mixture with Reclaimed Asphalt Pavement. Transportation Research Record, n. 2126, p. 109-114. DOI: $10.3141 / 2126-13$.

Leite, L. F. M. e I. A. Tonial (1994) Qualidade dos Cimentos Asfálticos Brasileiros Segundo as Especificações SHRP. Trabalhos Técnicos, Instituto Brasileiro de Petróleo, Gás e Biocombustíveis, Rio de Janeiro, p. 94-119.

Li, X.; T. Clyne; G. Reinke; E. N. Johnson; N. Gibson e M. E. Kutay (2011) Laboratory Evaluation of Asphalt Binders and Mixtures Containing Polyphosphoric Acid. Transportation Research Record, n. 2210, p. 47-56. DOI: 10.3141/2210-06.

Marasteanu, M. O.; T. Clyne; J. McGraw; X. Li e R. Velasquez (2005) High-Temperature Rheological Properties of Asphalt Binders. Transportation Research Record, n. 1901, p. 52-59. DOI: 10.3141/1901-07.

Moura, E. (2010) Estudo de Deformação Permanente em Trilha de Roda de Misturas Asfálticas em Pista e em Laboratório. Tese (Doutorado em Engenharia de Transportes) - Departamento de Engenharia de Transportes, Escola Politécnica da Universidade de São Paulo, São Paulo. DOI: 10.11606/T.3.2010.tde17082010-094223.

Nascimento, L. A. H. (2008) Nova Abordagem da Dosagem de Misturas Asfálticas Densas com Uso do Compactador Giratório e Foco na Deformação Permanente. Dissertação (Mestrado em Engenharia Civil) - Instituto Alberto Luiz Coimbra de PósGraduação e Pesquisa em Engenharia, Universidade Federal do Rio de Janeiro, Rio de Janeiro.

Onofre, F. C. (2012) Avaliação do Comportamento Mecânico de Misturas Asfálticas Produzidas com Ligantes Asfálticos Modificados por Ácido Polifosfórico e Aditivos Poliméricos, Enfatizando a Resistência à Deformação Permanente. Dissertação (Mestrado em Engenharia de Transportes) - 
Departamento de Engenharia de Transportes, Universidade Federal do Ceará, Fortaleza.

Onofre, F. C.; V. T. F. Castelo Branco; J. B. Soares e A. L. Faxina (2013) Avaliação do Efeito de Ligantes Asfálticos Modificados na Resistência à Deformação Permanente de Misturas Asfálticas Densas. Transportes, v. 21, n. 3, p. 14-21. DOI: 10.4237/transportes.v21i3.685.

Reinke, G. (2010) Use of Hamburg Rut Testing Data to Validate the Use of $\mathbf{J}_{\mathrm{nr}}$ as a Performance Parameter for High-Temperature Permanent Deformation. Transportation Research Circular, n. EC147, p. 14-24.

Roberts, F. L.; P. S. Kandhal; E. R. Brown; D.-Y. Lee e T. W. Kennedy (1996) Hot Mix Asphalt Materials, Mixture Design and Construction (2nd ed.). National Asphalt Pavement Association Research and Education Foundation, Lanham.

Wasage, T. L. J.; I. B. Kazatchkov; J. Stastna e L. Zanzotto (2009) Rutting Evaluation of Asphalt Binders and Mixes. In: Loizos, A.; M. Partl; T. Scarpas e I. L. Al-Qadi (eds.) Advanced Testing and Characterisation of Bituminous Materials. CRC Press/Balkema, Leiden, v. 2, cap. 7, p. 989-998.

DOI: 10.1201/9780203092989.ch95.

Wasage, T. L. J.; J. Stastna e L. Zanzotto (2011) Rheological Analysis of Multi-Stress Creep Recovery (MSCR) Test.

International Journal of Pavement Engineering, v. 12, n. 6, p. 561-568. DOI: 10.1080/10298436.2011.573557.

Witczak, M. W.; K. Kaloush; T. Pellinen; M. El-Basyouny e H. Von Quintus (2002) Simple Performance Test for Superpave Mix Design. NCHRP Report 465, Transportation Research Board, Washington, DC 